\title{
Soluções exatas de um modelo matemático de invasão tumoral
}

\author{
Altemir Bortuli Junior ${ }^{1}$ \\ Programa de Pós-Graduação em Matemática/UFABC, Santo André, SP \\ Igor Leite Freire ${ }^{2}$ \\ CMCC/UFABC, Santo André, SP \\ Norberto Anibal Maidana ${ }^{3}$ \\ CMCC/UFABC, Santo André, SP
}

\begin{abstract}
Resumo. Neste trabalho estudamos um modelo matemático de invasão tumoral. O modelo consiste em um sistema não-linear de equações diferenciais parciais que descreve a dinâmica de interações entre a densidade de células tumorais, a densidade da matriz extracelular, a concentração de enzimas degradantes da matriz e a concentração de oxigênio. Obtemos soluções exatas do modelo aplicando a técnica de simetrias de Lie.
\end{abstract}

Palavras-chave. Tumores Sólidos Localizados, Invasão de Tumores, Modelos Matemáticos, Simetrias de Lie, Soluções Exatas

\section{Introdução}

O câncer é uma das principais causas de morte em todo o mundo. A Agência Internacional de Pesquisa em Câncer (IARC), ligada a Organização Mundial da Saúde (OMS), estimou a incidência de 18, 1 milhões de casos e 9,6 milhões de mortes por câncer no mundo em 2018 [11]. A incidência e mortalidade aumentam com a idade, com aproximadamente $60 \%$ de todos os casos diagnosticados e $70 \%$ de todas as mortes por câncer ocorrendo em pessoas com 65 anos ou mais $[1,12]$.

Câncer é na verdade um termo genérico que descreve um grupo heterogêneo de doenças caracterizadas pelo crescimento descontrolado e disseminação de células anormais [14, 15]. As muitas diferenças entre os casos de câncer é uma das principais razões pelas quais o entendimento, o tratamento e a cura são tão difíceis. Essa diversidade reflete na dificuldade em desenvolver um modelo matemático (contínuo) unificado que descreva os mais variados tipos de câncer em todos os seus estágios, apesar dos esforços feitos ao longo dos anos, veja [2-6] onde o câncer é explorado por meio de modelos matemáticos e computacionais.

Um modelo de particular interesse para este trabalho é o proposto por Anderson [5], que considera quatro variáveis: densidade de células tumorais, densidade da matriz extracelular (MEC), concentração de enzimas degradantes da matriz (MDEs) e concentração de oxigênio, que denotaremos por $N, E, M$ e $C$, respectivamente. A relação entre essas variáveis é descrita pelo sistema

\footnotetext{
${ }^{1}$ altemirbortulijunior@hotmail.com.

2 igor.freire@ufabc.edu.br.

${ }^{3}$ norberto.maidana@ufabc.edu.br.
} 


$$
\left\{\begin{array}{l}
N_{t}=D_{1} \nabla^{2} N-\rho \nabla \cdot(N \nabla E), \\
E_{t}=-\delta M E, \\
M_{t}=D_{2} \nabla^{2} M+\mu N-\lambda M, \\
C_{t}=D_{3} \nabla^{2} C+\beta E-\gamma N-\alpha C,
\end{array}\right.
$$

em que $D_{1}, D_{2}, D_{3}, \rho, \delta, \mu, \lambda, \beta, \gamma$ e $\alpha$ são constantes não-negativas, $(.)_{t}=\partial_{t}(),. \nabla=\left(\partial_{x}, \partial_{y}, \partial_{z}\right)$ é o gradiente e $\nabla^{2}=\nabla$. $\nabla$ é o laplaciano em 3-dimensões. O domínio do sistema é uma região limitada do tecido, denotada por $\Omega$, com condições iniciais apropriadas para cada variável. Uma vez que as células tumorais e as MDEs permanecem, no estágio avascular, dentro do domínio, condições apropriadas de contorno também são impostas em $\partial \Omega$, fronteira de $\Omega$.

O sistema (1) descreve a degradação da ECM pelo contato com as MDEs, com uma constante de degradação $\delta$. Por outro lado, as MDEs são produzidas pelas células tumorais a uma taxa $\mu$ e decaem a uma taxa $\lambda$. Como as MDEs degradam a ECM, as células tumorais se espalham pelo tecido devido a uma combinação de difusão e haptotaxia, com coeficiente haptotático $\rho$. O oxigênio é produzido pela ECM a uma taxa $\beta$, espalha-se nela por difusão, é absorvido pelo tumor a uma taxa $\gamma$ e decai naturalmente a uma taxa $\alpha$. Os coeficientes de difusão das células tumorais, das MDEs e do oxigênio são $D_{1}, D_{2}$ e $D_{3}$, respectivamente.

O sistema (1), proposto em [5], surge do acréscimo da equação que descreve a dinâmica do oxigênio, essencial no processo de crescimento e invasão de tumores sólidos, ao sistema (5) proposto em [4]. A análise do sistema (5) de [4] é numérica e, até onde sabemos, as únicas soluções analíticas dele são obtidas em $[8,9]$ pela aplicação da técnica de simetrias de Lie $[7,13]$.

Neste trabalho, embasados pelos resultados apresentados em [9], determinamos soluções analíticas exatas do sistema (1) pela aplicação da técnica de simetrias de Lie.

\section{Soluções exatas com simetrias esféricas}

Uma vez que a equação que descreve a dinâmica do oxigênio no sistema (1) é invariante por rotações, lembrando que as demais equações do sistema (1) também o são [9], aplicamos a técnica de simetrias de Lie, veja [13], p.225, para construirmos os invariantes

$$
N=\Phi_{1}(r, t), E=\Phi_{2}(r, t), M=\Phi_{3}(r, t) \text { e } C=\Phi_{4}(r, t),
$$

em que

$$
r=\sqrt{x^{2}+y^{2}+z^{2}}
$$

Consequentemente, impondo que os invariantes (2) sejam soluções do sistema (1), reescrevemo-lo em coordenadas esféricas da seguinte forma:

$$
\left\{\begin{array}{l}
N_{t}=D_{1}\left(N_{r r}+\frac{2}{r} N_{r}\right)-\rho\left(N_{r} E_{r}+N E_{r r}+\frac{2}{r} N E_{r}\right), \\
E_{t}=-\delta M E, \\
M_{t}=D_{2}\left(M_{r r}+\frac{2}{r} M_{r}\right)+\mu N-\lambda M, \\
C_{t}=D_{3}\left(C_{r r}+\frac{2}{r} C_{r}\right)+\beta E-\gamma N-\alpha C .
\end{array}\right.
$$


Agora, consideramos o caso em que as MDEs não sofrem nenhuma forma de decaimento, o oxigênio não é produzido e nem decai naturalmente, ou seja, $\lambda=\beta=\alpha=0$ em (3). Para este caso, o teorema a seguir nos fornece informações necessárias para construção de soluções.

Teorema 2.1. Se $D_{1} D_{2} D_{3} \rho \delta \mu \gamma \neq 0$ e $\lambda=\beta=\alpha=0$, então o operador

$$
X=\frac{r}{2} \partial_{r}+t \partial_{t}-2 N \partial_{N}-M \partial_{M}-C \partial_{C}
$$

é um gerador de simetrias do sistema (3).

Uma vez que, sob essas restrições, o operador $X$ é um gerador de um grupo de simetrias a 1-parâmetro do sistema (3), podemos construir os invariantes

$$
N=\frac{1}{r^{4}} \Phi_{1}(w), E=\Phi_{2}(w), M=\frac{1}{r^{2}} \Phi_{3}(w) \text { e } C=\frac{1}{r^{2}} \Phi_{4}(w),
$$

em que $w=\frac{r}{\sqrt{t}}$.

Assim, considerando que os invariantes (4) sejam soluções do sistema (3), reescrevemo-lo da seguinte forma:

$$
\left\{\begin{aligned}
-\frac{1}{2} w^{3} \Phi_{1}^{\prime} & =D_{1}\left(12 \Phi_{1}-6 w \Phi_{1}^{\prime}+w^{2} \Phi_{1}^{\prime \prime}\right)-\rho\left(w^{2} \Phi_{1}^{\prime} \Phi_{2}^{\prime}+w^{2} \Phi_{1} \Phi_{2}^{\prime \prime}-2 w \Phi_{1} \Phi_{2}^{\prime}\right), \\
\frac{1}{2} w^{3} \Phi_{2}^{\prime} & =\delta \Phi_{3} \Phi_{2}, \\
-\frac{1}{2} w^{3} \Phi_{3}^{\prime} & =D_{2}\left(2 \Phi_{3}-2 w \Phi_{3}^{\prime}+w^{2} \Phi_{3}^{\prime \prime}\right)+\mu \Phi_{1}, \\
-\frac{1}{2} w^{3} \Phi_{4}^{\prime} & =D_{3}\left(2 \Phi_{4}-2 w \Phi_{4}^{\prime}+w^{2} \Phi_{4}^{\prime \prime}\right)+\gamma \Phi_{1} .
\end{aligned}\right.
$$

Em [9], para o sistema (5) na ausência da equação que descreve a dinâmica do oxigênio, é apresentada uma solução particular para o caso em que $D_{1}=D_{2}$. Logo, resta-nos determinar $\Phi_{4}$ para obter uma solução de (5). Entretanto substituindo $\Phi_{1}$, determinada em [9], na equação do oxigênio e resolvendo para $\Phi_{4}$, obtemos as duas seguintes soluções do sistema (5):

1. Se $D_{1}=D_{2}<D_{3}$, então

$$
\begin{aligned}
& \Phi_{1}(w)=w^{3} e^{-\frac{w^{2}}{4 D_{1}}}\left(w^{2}-2 D_{1}\right) \\
& \Phi_{2}(w)=0 \\
& \Phi_{3}(w)=\mu w^{3} e^{-\frac{w^{2}}{4 D_{1}}} \\
& \Phi_{4}(w)=\frac{4 \gamma D_{1}^{2} w e^{-\frac{w^{2}}{4 D_{1}}}}{D_{3}-D_{1}}
\end{aligned}
$$

2. Se $D_{1}=D_{2}=D_{3}$, então

$$
\begin{aligned}
& \Phi_{1}(w)=w^{3} e^{-\frac{w^{2}}{4 D_{1}}}\left(w^{2}-2 D_{1}\right), \\
& \Phi_{2}(w)=0 \\
& \Phi_{3}(w)=\mu w^{3} e^{-\frac{w^{2}}{4 D_{1}}}, \\
& \Phi_{4}(w)=\gamma w\left(1-w^{2}\right) e^{-\frac{w^{2}}{4 D_{1}}} .
\end{aligned}
$$


Também, considerando a solução obtida em [9], para o caso em que $D_{1} \neq D_{2}$, e repetindo o raciocínio feito acima, obtemos mais duas soluções para o sistema (5), que serão apresentadas no evento.

Por fim, destacamos que reportamos uma discussão mais ampla desses resultados em [10].

\section{Conclusões}

A aplicação da técnica de simetrias de Lie possibilitou a obtenção de soluções particulares do sistema (1), que descrevem um caso extremo em que o câncer degradou toda a ECM. Assim, a técnica tem se mostrado efetiva na obtenção de soluções exatas de modelos matemáticos de invasão tumoral.

\section{Agradecimentos}

O primeiro autor agradece à UFABC pelo apoio financeiro. O segundo autor agradece ao $\mathrm{CNPq}$ (processos 404912/2016-8 e 308516/2016-8) pelo auxílio financeiro.

\section{Referências}

[1] Al-Mansour, Z., Pang, L. and Bathini, V. Novel Cancer Therapeutics in Geriatrics: What is Unique to the Aging Patient?, Drugs \& aging, 36:1-11, 2019. DOI: 10.1007/s40266-018-0619-2.

[2] Byrne, H. M. Dissecting cancer through mathematics: from the cell to the animal model, Nat. Rev. Cancer, 10:221-230, 2010. DOI: 10.1038/nrc2808.

[3] Altrock, P. M., Liu, L. L. and Michor, F. The mathematics of cancer: integrating quantitative models, Nat. Rev. Cancer, 15:730-745, 2015. DOI: 10.1038/nrc4029.

[4] Anderson, A. R. A. et al.. Mathematical modelling of tumour invasion and metastasis, $J$. Theor. Med., 2:129-154, 2000.

[5] Anderson, A. R. A. A hybrid mathematical model of solid tumour invasion: the importance of cell adhesion, Mathematical medicine and biology: a journal of the IMA, 22:163-186, 2005. DOI: 10.1093/imammb/dqi005.

[6] Anderson, A. R. and Quaranta, V. Integrative mathematical oncology, Nat. Rev. Cancer, 8:227-234, 2008. DOI: 10.1038/nrc2329.

[7] Bluman, G. W., Cheviakov, A. F. and Anco, S. C. Applications of Symmetry Methods to Partial Differential Equations. Springer, New York, 2010.

[8] Bortuli Junior, A., Freire, I. L. e Maidana, N. A. Tumores Sólidos Localizados: Uma Abordagem Via Simetrias de Lie, Proceeding Series of the Brazilian Society of Computational and Applied Mathematics, volume 6, 2018.

[9] Bortuli Junior, A., Freire, I. L. e Maidana, N. A. Soluções de Modelos Matemáticos de Tumores Sólidos Via Simetrias de Lie, Proceeding Series of the Brazilian Society of Computational and Applied Mathematics, volume 7, 2020. DOI: 10.5540/03.2020.007.01.0438.

[10] Bortuli Junior, A., Freire, I. L. e Maidana, N. A. Group classification and analytical solutions of a radially symmetric avascular cancer model, Studies in Applied Mathematics, 1-29, 2021. DOI: $10.1111 /$ sapm.12416 
[11] Bray, F. et al. Global cancer statistics 2018: GLOBOCAN estimates of incidence and mortality worldwide for 36 cancers in 185 countries, CA: a cancer journal for clinicians, 68:394-424, 2018. DOI: 10.3322 /caac.21492.

[12] Dotan, E. Advancing Treatment Approach to the Older Patient with Cancer Through Clinical Trials Participation, Surgical Oncology Clinics, 26:719-728, 2017. DOI: 10.1016/j.soc.2017.05.010.

[13] Ibragimov, N. H. Elementary Lie group analysis and ordinary differential equations, John Wiley and Sons, United Kingdom, 1999.

[14] https://training.seer.cancer.gov/disease/cancer/. Acesso em: 13 de fev. de 2020.

[15] https://www.inca.gov.br/o-que-e-cancer. Acesso em: 13 de fev. de 2020. 